\title{
On the Effect of Functionalizer Chain Length and Water Content in Polyethylene/Silica Nanocomposites: Part I - Dielectric Properties and Breakdown Strength
}

\author{
I. L. Hosier, M. Praeger, A. F. Holt, A. S. Vaughan and S. G. Swingler \\ Electrical and Electronic Engineering Group, \\ University of Southampton, \\ Highfield, Southampton, \\ SO17 1BJ, U.K.
}

\begin{abstract}
A series of nanoparticles was prepared by functionalizing a commercial nanosilica with alkylsilanes of varying alkyl tail length, from propyl to octadecyl. By using a constant molar concentration of silane, the density of alkyl groups attached to each system should be comparable. The effect of chain length on the structure of the resulting nanosilica/polyethylene nanocomposites was examined and comparison with an unfilled reference system revealed that, other than through a weak nucleating effect, the inclusion of the nanosilica does not affect the matrix structure. Since water interacts strongly with applied electric fields, water was used as a dielectric probe in conjunction with dielectric spectroscopy to examine the effect of the nanofiller and its surface chemistry on the system. Sets of samples were prepared through equilibrating under ambient conditions, vacuum drying and water immersion. While the water content of the unfilled polymer was not greatly affected, the water content of the nanocomposites varied over a wide range as a result of water accumulation, in a range of states, at nanoparticle interfaces. The effect of water content on breakdown behavior was also explored and, in the unfilled polymer, the breakdown strength was found to depend little on exposure to water ( $13 \%$ reduction). In all the nanocomposites, the increased propensity for these systems to absorb water meant that the breakdown strength was dramatically affected ( $>66 \%$ reduction).
\end{abstract}

Index Terms - Nanotechnology, Dielectric Breakdown, Conductivity, Silica, Polyethylene, Water.

\section{INTRODUCTION}

NANOTECHNOLOGY includes a vast range of concepts spanning the development of nanoscale electronic devices to material systems whose macroscopic characteristics originate at the nanometre level. Nanocomposites can conveniently be defined as material systems that combine a matrix and a filler, where the latter exhibits at least one dimension that falls below $100 \mathrm{~nm}$ and where this results in some properties not exhibited by the individual components alone. While nanocomposites have been exploited empirically since Roman times [1], understanding of the underlying physics has lagged behind deployment and this is particularly true in the case of the use of nanocomposites in dielectric applications: nanodielectrics.

Although nanocomposites based upon carbon black and an elastomeric matrix have been exploited throughout much of the

Manuscript received on 22 December 2015, in final form XX Month 2016. twentieth century, current interest in the general nanocomposite concept is frequently attributed to pioneering research at Toyota in the 1990s [2, 3] in connection with the use of nanoclays as a means of improving the behavior of thermoplastic systems. Subsequently, much of the consequent work in this area has similarly focused on their advantageous mechanical and thermal properties (see, for example, refs $[4,5]$ ). However, since the seminal paper by Lewis in 1994 [6], interest in developing nanostructured dielectrics has grown dramatically, such that many industrial concerns are now seeking to develop commercial products based upon nanodielectrics. However, the corpus of work published over the last twenty years clearly indicates a very mixed picture, with many inconsistent and even contradictory results being reported.

In terms of nanosilica/polyethylene (PE) nanocomposites the subject of this study - the situation regarding their dielectric properties is far from clear. Since the attractive properties of nanodielectrics have generally been ascribed to the large 
specific interfacial area that exists within such systems and the subsequent formation of interphases [6, 7], achieving good nanoparticle dispersion and avoiding particle aggregation is generally considered to be key, but is very difficult to achieve consistently [8-12]. Consequently, when the breakdown strength of a nanocomposite is compared with that of an identical polymer without nanofiller, examples can be found where the addition of the nanofiller reduced the breakdown strength [13, 14], where no change in breakdown strength was seen [15] or where increased breakdown strength occurred [16, 17]. A possible explanation for these apparent contradictions may be found in morphological data (where this is provided); poor nanofiller dispersion seems to lead to detrimental effects on breakdown strength $[10,11,18]$ whilst well dispersed systems seem to show improved performance $[15,16]$. Poor nanoparticle dispersion and particle aggregation is a particular issue at high filler loadings, leading to poor breakdown strength even in otherwise well dispersed systems [15].

Only a few reports exist where the electrical conductivity of a nanosilica/polymer composite is compared with the identical polymer without nanoparticles present $[5,8,19,20]$. Whilst three of these reports indicate an increase in electrical conductivity when the nanofiller is added $[8,19,20]$, the other indicates, in better dispersed systems, a reduction [5]. Again, this apparent contradiction may be explained through variations in nanofiller dispersion; Veena et al. [5] make the important point, that for high filler loadings where dispersion is poor, this leads directly to increased conductivity.

Dielectric spectroscopy findings are also somewhat inconsistent. Whilst the addition of a nanofiller leads to two characteristic loss peaks and an increase in permittivity at low frequencies $[8,9,12,16,18,21,22]$, in cases where the nanofiller is well dispersed, this is not observed. Instead, the dielectric loss increases in a similar manner to that in comparable micro-filled systems [15, 16-17, 20]. Such behavior can be explained by changes in nanofiller dispersion - poorly dispersed nanoparticles lead to increased dielectric loss since, then, they effectively behave as micrometric inclusions. However this is not the only variable, since dielectric data are also strongly influenced by water content; the associated loss peaks tend to shift towards higher frequencies and the permittivity increases in wetter samples [12, 18, 21, 22].

The above review of the available literature, whilst by no means exhaustive, nevertheless clearly highlights the inconsistent nature of published nanodielectrics research. While it is tempting to ascribe such effects to subtle variations in the materials used by different researchers, different processing conditions, etc., including the impact of such factors on nanoparticle dispersion, the final point raises an additional issue worthy of consideration, namely, environmental factors. Water is ubiquitous and it is well known that measured dielectric properties are sensitive to absorbed water [12, 18, 21, 22]. Many researchers have already highlighted the crucial role of the nanoparticle/polymer interface $[6,8,16-18,21-23]$ and surface functionalization $[11,12,16,17,24]$ in determining dielectric properties. Consequently, it is easy to envisage how, for a hydrophilic system such as nanosilica, the nanofiller/matrix interface can be influenced by absorbed water and, consequently, the potentially critical role of surface functionalization in limiting or promoting water uptake.

The investigation reported here therefore set out with two distinct objectives: (1) to investigate the influence of surface chemistry on the structure and dielectric response of polyethylene/nanosilica nanocomposites, where alkyl chains of different length (from propyl to octadecyl) had been attached using silane chemistry in order to render the nanofiller and matrix more compatible, (2) to consider, in detail, the influence of absorbed water on the dielectric response of these systems. In this paper, we consider the effect of the different nanosilicas on the structure of the matrix polymer and report on water uptake, dielectric relaxation and DC breakdown behavior. The accompanying publication reports on DC conduction and space charge in the same systems and draws global conclusions.

\section{EXPERIMENTAL}

\subsection{MATERIALS}

Three silane functionalizing agents were employed, with varying alkyl tail lengths ranging from 3 to 18 carbon atoms: trimethoxy(propyl)silane (97\% purity); trimethoxyl(octyl)silane (96\% purity); trimethoxy(octadecyl)silane (90 $\%$ purity). Some of their physical properties (from manufacturers' data sheets) are listed in Table 1. The nanofiller was silicon dioxide $\left(\mathrm{SiO}_{2}\right)$ with a quoted particle size of 10-20 nm; all four materials were obtained from Sigma Aldrich and were used as supplied. The high density polyethylene (HDPE) was Rigidex HD5813EA (BP Chemicals), whilst the low density polyethylene (LDPE) was LD100BW (ExxonMobil Chemicals).

\subsection{FUNCTIONALIZATION OF NANOSILICA}

The as-supplied nanosilica was functionalized using an anhydrous route [25]. The advantage of anhydrous functionalization compared to the more traditional alcohol/water route is that reactions are largely confined to the hydroxyl groups located on the surfaces of the nanosilica particles, such that silane-silane reactions are suppressed [11]. To ensure that the extent of surface functionalization was broadly equivalent across all three

Table 1. Physical structure and data for the three functionalizing agents used in this study

\begin{tabular}{|c|c|c|c|c|}
\hline Functionalizer & Physical structure & Molar mass (g/mol) & Density (g/ml) & Amount used (ml) \\
\hline Trimethoxy(propyl)silane & $\mathrm{Si}-\left(\mathrm{OCH}_{3}\right)_{3}-\left(\mathrm{CH}_{2}\right)_{2}-\mathrm{CH}_{3}$ & 164.3 & 0.932 & 4.77 \\
\hline Trimethoxyl(octyl)silane & $\mathrm{Si}-\left(\mathrm{OCH}_{3}\right)_{3}-\left(\mathrm{CH}_{2}\right)_{7}-\mathrm{CH}_{3}$ & 234.4 & 0.907 & 7.00 \\
\hline Trimethoxyl(octadecyl)silane & $\mathrm{Si}-\left(\mathrm{OCH}_{3}\right)_{3}-\left(\mathrm{CH}_{3}\right)_{17}-\mathrm{CH}_{3}$ & 374.7 & 0.883 & 11.49 \\
\hline
\end{tabular}


functionalizing agents, a constant molar concentration was used here, as indicated in Table 1. In previous work [11, 24], a fixed $7 \mathrm{ml}$ of functionalizing agent was used to functionalize $15 \mathrm{~g}$ of nanosilica, irrespective of the silane, such that variations in the silane molar mass will result in changes in the degree of surface functionalization.

Here, $15 \mathrm{~g}$ of nanosilica were dispersed in $200 \mathrm{ml}$ of dry tetrahydrofuran (THF) and the required amount of silane was added. The mixture was sonicated for $10 \mathrm{~min}$, stirred in a sealed rotary evaporator at $80 \mathrm{rpm}$ for $48 \mathrm{~h}$ and centrifuged at $3500 \mathrm{rpm}$ for $10 \mathrm{~min}$ before the supernatant was decanted off. Fresh THF was added and the system was vigorously stirred until the nanosilica was again suspended. This washing process was repeated twice with THF and twice with diethyl ether. The residual solvent was removed using a rotary evaporator and the resulting white powder was finally vacuum dried at room temperature for $24 \mathrm{~h}$ before being stored in a desiccator. Functionalization was verified by $\mathrm{CHN}$ analyzis (Medac Ltd) of the unfunctionalized and functionalized nanosilicas. While the carbon content of the former was found to be $0.28 \pm 0.02$ $\%$, all functionalized systems gave values in excess of $5 \%$. Elsewhere [17], similarly processed nanosilicas revealed evidence of $\mathrm{CH}_{2}$ groups in the Fourier infra-red spectrum, further supporting the efficacy of the methodology.

\subsection{BLENDING}

A blend composed of $20 \%$ HDPE and $80 \%$ LDPE was used as the base resin, due to the clarity with which changes in matrix morphology are revealed when isothermally crystallized [26]. A control sample (no nanosilica) was prepared identically and will be referred to as CT1. Three other blends incorporating 10 wt. $\%$ of nanosilica, functionalized using the various functionalizing agents, will be referred to NS3, NS8 or NS18, where the numeral indicates the number of carbon atoms in the attached alkyl chain, as specified in Table 2.

Solution blends were prepared in $10 \mathrm{~g}$ amounts using xylene as the solvent and methanol as the non-solvent. First, the required amount ( $1 \mathrm{~g})$, of the treated nanosilica was placed into $200 \mathrm{ml}$ of xylene and the resulting mixture was sonicated for $1 \mathrm{~h}$ to form a suspension. The polymer was then added (1.8 $\mathrm{g}$ HDPE and 7.2 $\mathrm{g}$ LDPE) and the mixture was brought to the boil whilst stirring vigorously. After $20 \mathrm{~min}$, the polymer and dispersed nanosilica were precipitated by pouring directly into $200 \mathrm{ml}$ of cold methanol and separated from the solvent by filtration. The resulting white solid was vacuum dried at $60{ }^{\circ} \mathrm{C}$ for $24 \mathrm{~h}$ and then pressed at $160{ }^{\circ} \mathrm{C}$ into $1 \mathrm{~mm}$ thick sheets.

\subsection{SAMPLE PREPARATION AND CONDITIONING}

Samples for characterisation were prepared in the form of $0.2 \mathrm{~mm}$ and $0.1 \mathrm{~mm}$ thick sheets using a hydraulic press and a steel mould with appropriate Melinex spacers $\left(160^{\circ} \mathrm{C}\right.$ and 3 ton load). All samples were crystallized from the melt for $1 \mathrm{~h}$ in an oil bath maintained at $115^{\circ} \mathrm{C}$, prior to quenching in water [26]. Following sample preparation and the application of gold electrodes as required, specimens were conditioned, to vary their water content, as follows: ambient samples were maintained under ambient conditions in an air conditioned environment $\left(19 \pm 2{ }^{\circ} \mathrm{C}, 55-80 \% \mathrm{RH}\right)$ for at least $14 \mathrm{~d}$ prior to testing; dry samples were vacuum dried at room temperature for periods of up to $14 \mathrm{~d}$; wet samples were immersed in water for periods of up to $14 \mathrm{~d}$. To determine the extent of water absorption, $0.2 \mathrm{~mm}$ thickness plaques of each material (total mass $\sim 0.5 \mathrm{~g}$ ) were dried or wetted at the same time and during this process their mass was recorded at regular intervals to an accuracy of $\pm 0.0001 \mathrm{~g}$ (i.e. $\pm 0.02 \%$ ).

\subsection{CHARACTERISATION}

Thermogravimetric analyzis (TGA) was performed by heating $5 \mathrm{mg}$ samples using a Perkin Elmer Pyris 1 TGA at a rate of $20 \mathrm{~K} / \mathrm{min}$ in air. Samples $2-5 \mathrm{mg}$ in size were subjected to analyzis by differential scanning calorimetry (DSC) - a Perkin Elmer DSC-7 instrument was used, which was routinely calibrated using high purity indium. Melting scans were obtained by heating at $10 \mathrm{~K} / \mathrm{min}$, whereas crystallization behavior was inferred by cooling at $5 \mathrm{~K} / \mathrm{min}$ from the melt.

Samples for scanning electron microscopy (SEM) were etched for $4 \mathrm{~h}$ in a solution of $1 \%$ potassium permanganate in an acid mixture (5:2:1 of sulphuric acid, phosphoric acid and water respectively) according to published procedures [27]. Samples were then mounted onto aluminium SEM stubs, gold coated and examined at $15 \mathrm{kV}$ in a JEOL JSM6500F high resolution FEG-SEM.

Dielectric spectroscopy was performed on $0.1 \mathrm{~mm}$ thick samples incorporating gold-coated $30 \mathrm{~mm}$ diameter electrodes, using a parallel plate test cell with a guard ring (diameter of inner electrode $30 \mathrm{~mm}$ ). Measurements of complex permittivity at room temperature were performed from $0.1 \mathrm{~Hz}$ to $100 \mathrm{kHz}$ using a Solartron 1296 dielectric interface linked to a Schlumberger SI 1260 impedance-gainphase analyzer.

DC breakdown testing was carried out on uncoated $0.1 \mathrm{~mm}$ thick samples placed between opposing $6.3 \mathrm{~mm}$ diameter ball bearing electrodes in a bath of silicone fluid (Dow Corning 200/20CS). An increasing DC voltage

Table 2. Blends used in these investigations

\begin{tabular}{c|c|c}
\hline Blend & Base Resin & Nano-filler used \\
\hline CT1 & $20 \% \mathrm{HDPE} / \mathrm{LDPE}$ & - \\
NS3 & $20 \% \mathrm{HDPE} / \mathrm{LDPE}$ & $10 \%$ nSi functionalized with trimethoxy(propyl)silane \\
NS8 & $20 \% \mathrm{HDPE} / \mathrm{LDPE}$ & $10 \%$ nSi functionalized with trimethoxy(octyl)silane \\
NS18 & $20 \% \mathrm{HDPE} / \mathrm{LDPE}$ & $10 \%$ nSi functionalized with trimethoxy(octadecyl)silane \\
\hline
\end{tabular}


Table 3. TGA, water absorption and DSC data

\begin{tabular}{|c|c|c|c|c|c|c|c|c|}
\hline & \multicolumn{3}{|c|}{ TGA data } & \multicolumn{2}{|c|}{ Water absorption } & \multicolumn{3}{|c|}{ DSC data } \\
\hline & $M_{\text {init }}(\%)$ & $T_{0.5}\left({ }^{\circ} \mathrm{C}\right)$ & $R_{570}(\%)$ & $L \max (\%)$ & $G_{\max }(\%)$ & $T_{m}\left({ }^{\circ} \mathrm{C}\right)$ & $T_{c}\left({ }^{\circ} \mathrm{C}\right)$ & $\Delta H(\mathrm{~J} / \mathrm{g})$ \\
\hline CT1 & - & 411 & - & - & - & $104.7,124.6$ & $95.0,110.2$ & 102 \\
\hline NS3 & 0.9 & 442 & 8.9 & -0.53 & +0.82 & $104.9,124.1$ & $95.2,111.4$ & 87 \\
\hline NS8 & 1.1 & 431 & 9.5 & -0.44 & +0.72 & $104.5,124.5$ & $94.7,112.0$ & 92 \\
\hline NS18 & 1.1 & 437 & 9.1 & -0.37 & +0.70 & $104.7,124.7$ & $94.6,111.8$ & 93 \\
\hline
\end{tabular}

$(100 \mathrm{~V} / \mathrm{s})$ was applied to this arrangement until failure occurred. For each system, 20 such tests were carried out, each at a separate location on the sample, the ball bearings being replaced after every 10 tests. The resulting data were then analyzed assuming Weibull statistics.

\section{RESULTS}

\subsection{THERMOGRAVIMETRIC ANALYZIS (TGA)}

TGA data obtained from the unfilled reference, CT1, indicate that it starts to degrade at $300{ }^{\circ} \mathrm{C}$, reaching $50 \%$ of the initial mass at $410{ }^{\circ} \mathrm{C}\left(T_{0.5}\right.$ in Table 3$)$ and zero mass at $570{ }^{\circ} \mathrm{C}$. The nanocomposites all behave in a similar fashion: first, the samples lose $\sim 1 \%$ mass (possibly water) above $100{ }^{\circ} \mathrm{C}\left(M_{\text {init }}\right.$ in Table 3); $50 \%$ mass is reached at $\sim 440{ }^{\circ} \mathrm{C}$ (thus the composites are somewhat more thermally stable than the unfilled reference material); the samples then continue to lose mass up to $570{ }^{\circ} \mathrm{C}$. Taking this to be the point at which the polymer is completely degraded, the filler content of the composites ( $R_{570}$ in Table 3 ) is around $9 \%$, which is as expected, anticipating some loss during sample preparation and also some water in the starting powder.

\subsection{WATER ABSORBTION AND DESORPTION}

Figure 1 shows the variation of sample mass as a function of time during conditioning. All these data have been normalized with respect to the mass of the specimen, as initially determined at equilibrium under ambient conditions. For both dry and wet conditioning, the variation in the mass of the unfilled system is within the uncertainties in the measurements $( \pm 0.02 \%)$, indicating that a negligible change in water content occurs either in vacuum or through immersion in water. In the case of the nanocomposites, the data asymptotically approach a constant value (after $>7 \mathrm{~d}$ ) and, so, exponential curve fitting was used to evaluate the mass-loss asymptote resulting from vacuum drying, $\mathrm{L}_{\max }$, and mass-gain asymptote resulting from water immersion, $\mathrm{G}_{\max }$. The value of each of these quantities for each material system is presented in Table 3, which is consistent with data reported elsewhere for similar nanocomposite systems [12, 18]. Crucially, these results clearly demonstrate that nanocomposites stored under ambient conditions contain a significant quantity of water.

From Table 3 and Figure 1, NS3 is capable of losing or gaining significantly more water than NS8 or NS18, which both incorporate longer chain functionalizing agents. All three systems were formulated using the same number of moles of silane so, assuming that the length of the aliphatic tail does not greatly influence the reactivity with nanosilica, the extent of surface functionalization should be broadly equivalent across all three functionalizing agents. The subtle effects of silane chain length seen in Figure 1 may therefore be related to reduced steric hindrance in the NS3 system permitting greater access of water to the surface of the nanoparticles [24]. The time constant of drying or wetting is independent of functionalizer chain length, $8 \pm 1 \mathrm{~d}$ for drying and $5 \pm 1 \mathrm{~d}$ for wetting so, under the conditions used here, removal of water from the initial, equilibrated systems occurs less readily than does water ingress.

\subsection{DIFFERENTIAL SCANNING CALORIMETRY}

DSC parameters obtained from the unfilled polyethylene blend and the three nanocomposites are listed in Table 3. In each system, two melting peaks ( $T_{m}$ in) are present; the lower occurs at $\sim 105{ }^{\circ} \mathrm{C}$ (enthalpy $\sim 55 \mathrm{~J} / \mathrm{g}$ ) and corresponds to the LDPE component of the blend, whilst the upper is at $\sim 124{ }^{\circ} \mathrm{C}$ (enthalpy $\sim 40 \mathrm{~J} / \mathrm{g}$ ) and corresponds, primarily, to the HDPE [26]. As expected, the total enthalpy $(\Delta H$ in Table 3) falls by $\sim 10 \%$ in the nanocomposites due to the presence of the filler. While the melting behavior is largely unaffected by the presence of the nanosilica, crystalliz ation (Figure $2 b$ ) of the HDPE phase occurs slightly earlier (112 ${ }^{\circ} \mathrm{C}, T_{c}$ in Table 3) in the nanocomposites compared to CT1 $\left(110^{\circ} \mathrm{C}\right)$, indicating a subtle nucleating effect of all three types of nanosilica on the polymer [4].

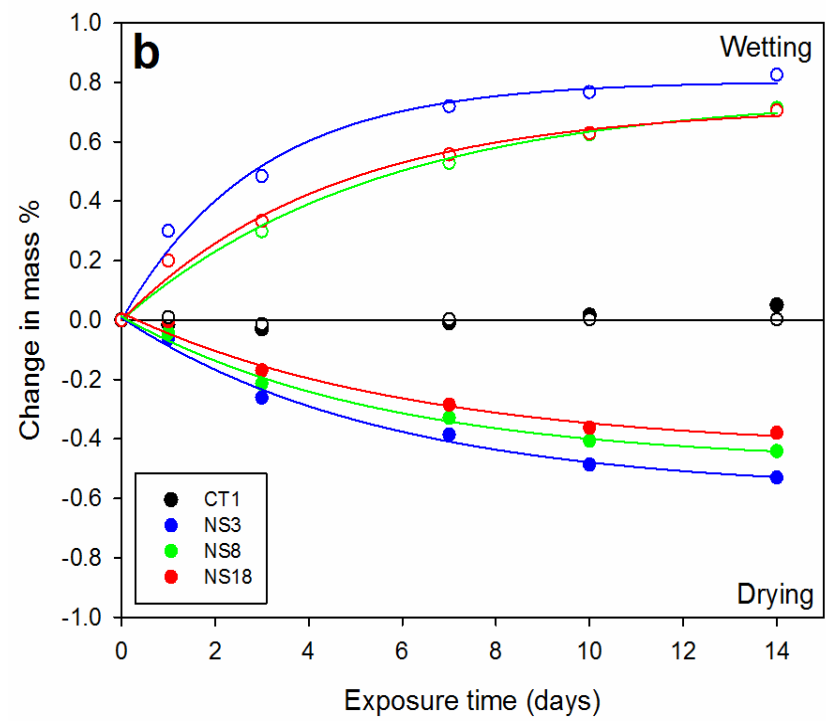

Figure 1. Measurements of water content (relative to ambient) for wet and dry conditioning with asymptotic fitted lines 

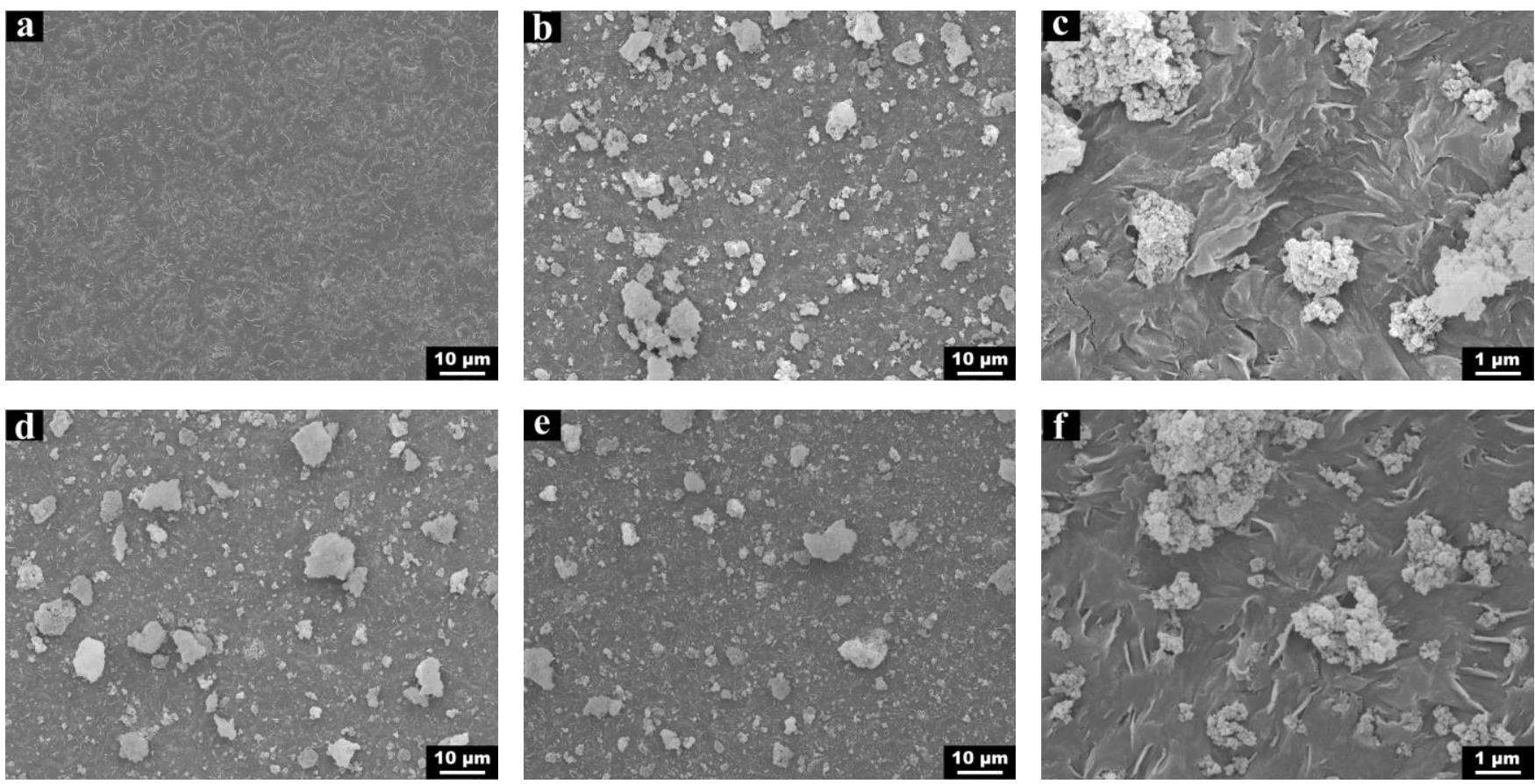

Figure 2. Representative SEM images comparing the typical morphology of systems considered here following isothermal crystallization at $115^{\circ} \mathrm{C}$ : (a) the typical banded spherulitic morphology of unfilled CT1; (b) low magnification image of NS3; (c) high magnification detail from NS3; (d) low magnification image of NS8; (e) low magnification image of NS18; (f) high magnification detail from NS18.

\subsection{MORPHOLOGY}

A selection of representative SEM micrographs is shown in Figure 2. CT1 exhibits a well-developed banded spherulitic morphology (Figure 2a), which is typical of polyethylene blends of the composition considered here following isothermally crystallization at $115^{\circ} \mathrm{C}[11,12,26]$. In all the nanocomposites, this underlying morphology is disrupted by the nanosilica as a consequence of (a) enhanced nucleation, as suggested by the DSC data, and (b) disrupted crystal growth. Figure $2 b$ shows a low magnification SEM image of NS3, from which it is evident that the nanosilica is agglomerated into structures up to $10 \mu \mathrm{m}$ in size. Whilst such aggregations are expected from this non-ideal sample preparation route and the high filler loading $[5,8,12,15]$, closer inspection reveals that these larger structures are composed of much smaller particles, with a dimension much closer to that specified by the supplier (Figure 2c); these large aggregates are clearly not completely broken up by the sonication process. NS8 and NS18 exhibit very similar morphologies (Figures 2d to 2f). Overall, varying the functionalizer chain length appears to have little influence on the observed morphologies and SEM examination of the as-supplied nanosilica confirms that it contains the aggregated structures shown above.

\subsection{DIELECTRIC SPECTROSCOPY}

Dielectric spectroscopy results are shown in Figures 3 and 4 as plots of the real $\left(\varepsilon^{\prime}\right)$ and imaginary parts of the relative permittivity $(\varepsilon ")$ respectively. In both figures, the dielectric response of CT1 (Figures 3a and 4a) appears independent of drying or water immersion, confirming that this unfilled sample is unaffected by either conditioning procedure. The real part of the relative permittivity is measured as $2.1 \pm 0.2$ (Figure 3a) while the imaginary part is $<0.01$ (Figure $4 \mathrm{a}$ ), values that are dominated by uncertainties in measuring the sample thicknesses and instrumental noise respectively. Considering first the real part of the relative permittivity, it is evident from Figure 3 that all nanocomposites behave in an identical manner, which is very different from that of the unfilled polyethylene. This indicates that the effects described below are related to the inclusion of the nanosilica. In the samples containing the least water $(14 \mathrm{~d}$ dried $)$, the most evident feature is a slight upturn in real permittivity at low frequencies. Then, as the moisture content increases, the value of $\varepsilon$ ' at low frequencies becomes progressively higher, with the upturn progressively shifting to higher frequencies. This is indicative of the presence of an increasing amount of polarizable material (i.e. water) in the system $[12,18,21$, 22], in agreement with Figure 1. Examining the effect of functionalizer chain length, no major statistically significant differences are evident. A previous study [12] of the effect of water absorption on polyethylene/silica nanocomposites reported comparable behavior to that described above. Specifically, for nanocomposites containing $10 \%$ nanosilica, $\varepsilon$ ' was found to tend asymptotically towards values of $\sim 6.8$ and $\sim 4.0$ for unfunctionalized and propylfunctionalized nanosilica respectively, after prolonged immersion in water. This dependence on nanosilica surface chemistry (hydroxyl in the unfunctionalized system, hydroxyl/alkyl after functionalization) indicates that water uptake occurs through adsorption at nanosilica surfaces. The value of $\varepsilon^{\prime}=4.5$ for the propyl-functionalized system considered here is therefore intermediate between the above two values. This reflects the fact that Lau et al. [12] used 

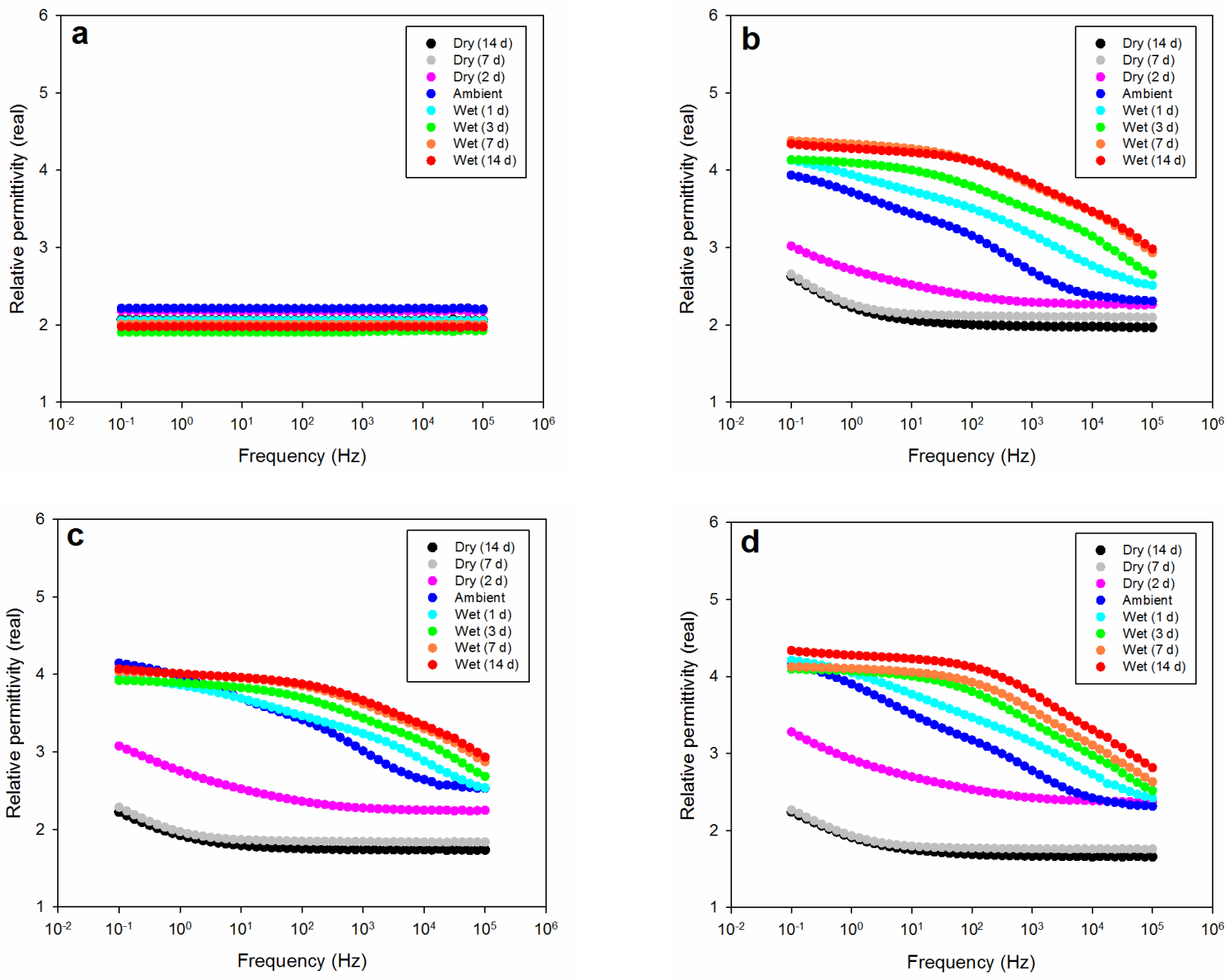

Figure 3. Plots showing the variation in the real part of the relative permittivity with frequency as a function of sample conditioning for; (a) CT1, (b) NS3, (c) NS8 and (d) NS18.

$\sim 50 \%$ more trimethoxy(propyl)silane to functionalize their nanosilica than in the work reported here, which results in increased alkyl surface character and, consequently, the absorption of less water.

Consider now the imaginary permittivity results shown in Figure 4. Taking all of these together, it is convenient to discuss them in terms of two broad relaxation peaks which, for the ambient nanocomposite samples, equate to a minor peak at $\sim 1 \mathrm{~Hz}$ and a major peak at $\sim 1 \mathrm{kHz}$. This behavior is very different from that seen in the case of the unfilled polyethylene, which indicates that the causal effects are again a consequence of the inclusion of the nanoparticles [8, 9]. With increasing water content (dried $\rightarrow$ ambient $\rightarrow$ wet samples), the major peak shifts from $<0.1 \mathrm{~Hz}$ to $100 \mathrm{kHz}$ and also increases somewhat in magnitude from 0.22 to 0.32 ; the minor peak also shifts to higher frequencies in a similar manner. This indicates that increasing the water content does not simply increase the strength of the associated relaxation also results in less constrained interfacial processes. These data are entirely consistent with the water uptake data presented in Figure 1.
Comparing the effect of the different functionalizer chain lengths, there are no significant differences in the dried samples although, in the ambient samples, an increase in the intensity of the low-frequency relaxation peak at $\sim 2 \mathrm{~Hz}$ is evident in NS18 (arrowed in Figure 4d). For water immersion times of $3 \mathrm{~d}$ and above, this then translates into a stronger and broader relaxation that spans the region 1$100 \mathrm{kHz}$. This suggests that a longer chain functionalizer increases the mobility of interfacial water structures despite a reduction in the overall amount of water absorbed (Table $3)$. That is, the presence of the functionalizer affects the interactions that occur between the water molecules and the nanoparticle surface [16, 23, 28, 29].

In the work of Lau et al. [12], the imaginary permittivity data of the dried sample containing 10\% of propylfunctionalized nanosilica was reported to be characterized by just one low frequency relaxation, as for all the samples shown in Figure 5 that had been dried for $14 \mathrm{~d}$. However, while the data shown in Figure 5 demonstrate that exposure to water ultimately results in the presence of high frequency relaxations in the range $1-100 \mathrm{kHz}$, such high frequency 

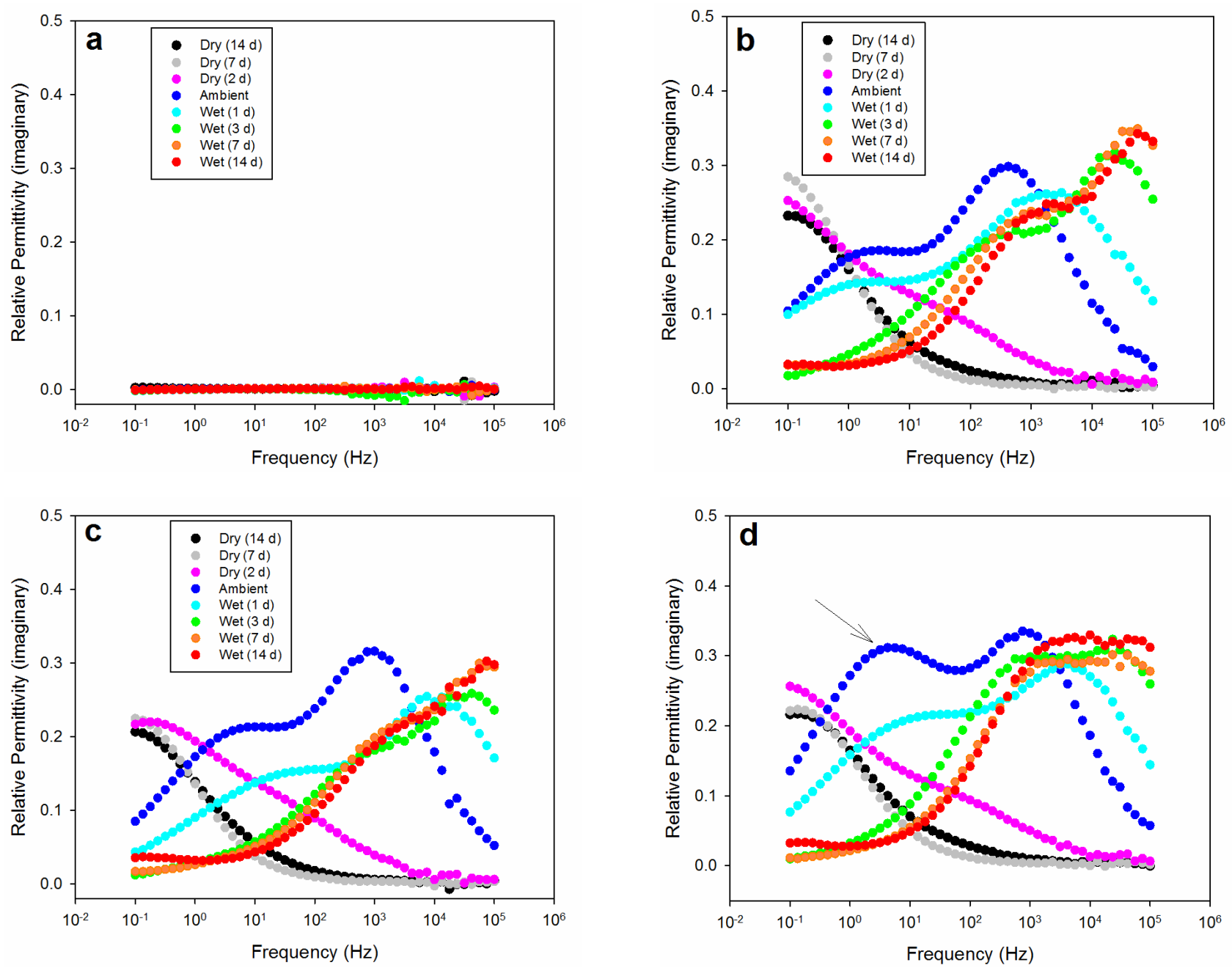

Figure 4. Plots showing the variation in the imaginary part of the relative permittivity with frequency as a function of sample conditioning for; (a) CT1, (b) NS3, (c) NS8 and (d) NS18 (legend omitted for clarity).

processes were only reported by Lau et al. [12] in the case of systems containing the unfunctionalized nanosilica. Presumably, this is again a reflection of the lower level of functionalization used in the study reported here.

The variation in the relaxation behavior seen in Figure 5 with water absorption can be explained in terms of the local conformation of water molecules at the nanoparticle/matrix interface, which will be influenced by the local structure of the material and, hence, the surface chemistry of the nanoparticle. As such, the water can be seen as a dielectric probe of the local interfacial structure, which is not easily examined directly by dielectric spectroscopy in the case of non-polar systems such as polyethylene. Steeman and Maurer [30] analyzed the case of a composite containing three phases, namely particles surrounded by an interphase embedded within a matrix. In the case of a conducting interphase, which they specifically evaluated in terms of a water shell around glass beads within a polyethylene matrix, the relaxation peak associated with the water shell is found to increase from $0.1 \mathrm{~Hz}$ to $50 \mathrm{kHz}$ with increasing volume fraction and/or conductivity of the interphase. Although this analysis does not correspond precisely with the circumstances pertinent to the data presented in Figure 5, we nevertheless believe that significant parallels exist. Initially, the water adsorbed onto the nanoparticles will be relatively strongly bound so, both dimensionally and in in terms of its mobility, it would be expected to manifest itself as a low frequency relaxation. As the quantity of water increases, the effective thickness of the aqueous water layer and the mobility of the molecules within it will increase, such that the relaxation moves progressively to high frequencies. In the work of Steeman and Maurer [30] this occurs continuously, whereas the data shown in Figure 5 is suggestive of at least two distinct processes that may reflect different aggregation states of water with different constraints; the Debye relaxation of free water should be observed at frequencies around $10 \mathrm{GHz}$ [18]. Hui et al. [18], however, assumed only one type of bound water, albeit that this paper only reports the dielectric response of dry and wet (i.e. saturated) specimens and, therefore, does not consider the possibility of intermediate scenarios.

From the above, absorption of water occurs readily in 

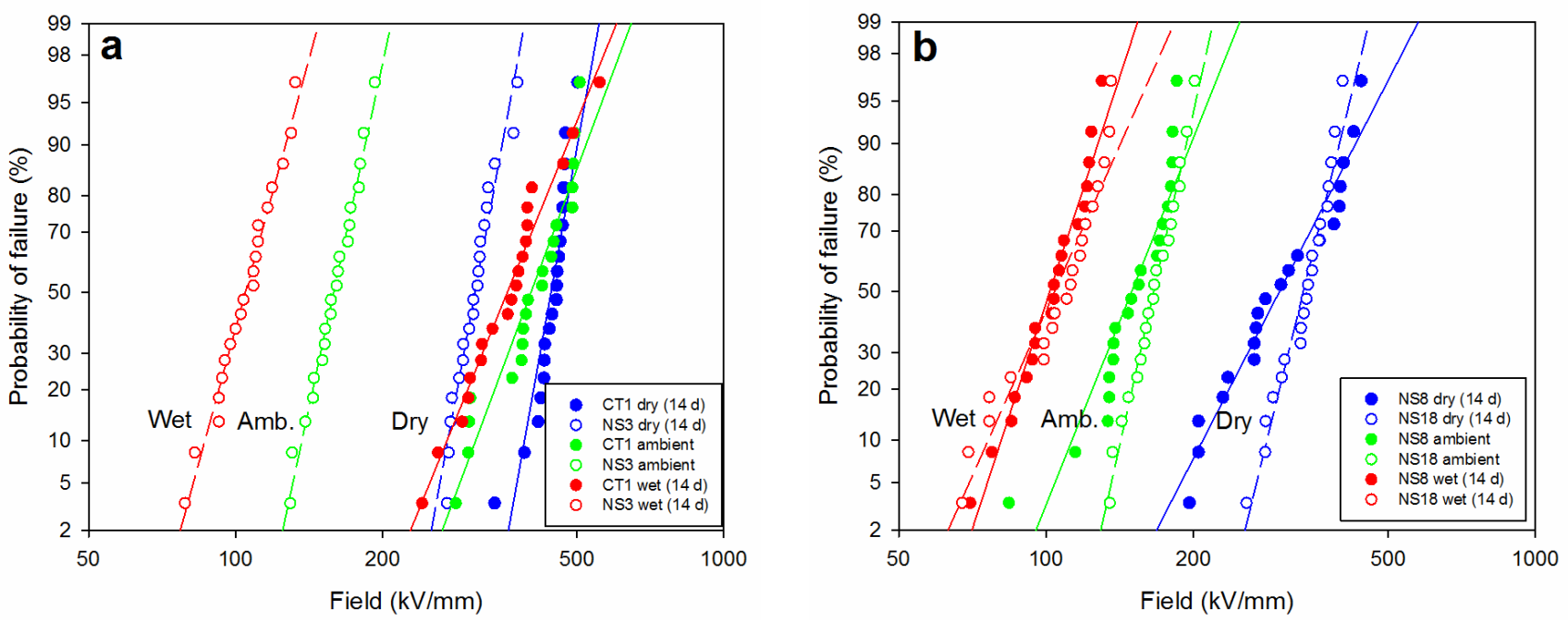

Figure 5. Weibull plots generated from DC breakdown data obtained from: (a) CT1 (closed symbols) and NS3 (open symbols); (b) NS8 (closed symbols) and NS18 (open symbols).

nanocomposites, even as a simple consequence of storage under ambient conditions and, in the case of the polyethylene matrix considered here, this results in an accumulation of water at nanoparticle surfaces. In view of the significance placed upon interfaces and interphases in determining the electrical behavior of nanodielectrics, it is pertinent to explore the effect of this water on an important characteristic, namely breakdown strength. For this, three systems have been chosen: specimens stored in vacuum for $14 \mathrm{~d}$, where Figure 1 indicates a minimum of water and, from the dielectric data, only a low frequency relaxation is present (Figure 4); ambient, where dielectric relaxations occur at intermediate frequencies; $14 \mathrm{~d}$ immersion, where the samples appear, effectively, to be saturated with water.

\subsection{BREAKDOWN}

DC breakdown data in the form of Weibull plots are shown in Figure 6. Although it would seem from the water immersion and dielectric data shown above that water absorption is negligible in CT1, the breakdown behavior indicates that this is not the case. In moving from the dried specimen through the ambient specimen to the sample subjected to $14 \mathrm{~d}$ of water immersion, the breakdown strength $\left(E_{o}\right)$ as represented by the Weibull scale parameter, progressively drops (see Table 4), albeit that the difference between the first two of these is not statistically significant. The implication of this is that DC breakdown is extremely sensitive to even small fluctuations in the water content of the specimen and, therefore, that for identical specimens, small differences in pre-conditioning (e.g. environmental humidity) can lead to significant differences in apparent breakdown strength. This point is reinforced when the various nanocomposites are considered. In all cases, desiccation or immersion in water leads to a much greater variation in water content than in CT1 which, in turn, manifests itself in a much greater variation in breakdown strength. Consequently, these data can, broadly, be divided into three groups, corresponding to dry, ambient and wet, as indicated on the plots. In the case of both the ambient samples and those tested after $14 \mathrm{~d}$ immersion, varying the silane coupling agent has a negligible effect on breakdown strength. However, in the case of the samples that were tested after prolonged drying, increasing the length of the alkyl tail from propyl to octyl to octadecyl results in a progressive increase in the Weibull scale parameter from $325 \mathrm{kV} \mathrm{mm}^{-1}$ to $355 \mathrm{kV} \mathrm{mm}^{-1}$. Since the uncertainty in these measurements is $\sim 20 \mathrm{kV} \mathrm{mm}{ }^{-1}$, this variation is not, strictly, of statistically significance. However, its monotonic nature combined with the fact that similar variations have been reported elsewhere [24] suggest that it may reflect a minor, but real, effect.

There are few studies in the literature that focus on the effect of water on the electrical breakdown strength of polyethylene. Nevertheless, one notable study on LDPE found a decrease in breakdown strength in samples subjected to humid conditions relative to dried ones [31]; whilst the behavior was largely attributed to enhanced space charge injection in the wet samples, the authors also made

Table 4. DC breakdown data

\begin{tabular}{|c|c|c|c|c|c|c|}
\hline & \multicolumn{2}{|c|}{ Dry (14 d) conditioned } & \multicolumn{2}{|c|}{ Ambient conditioned } & \multicolumn{2}{|c|}{ Wet $(14 \mathrm{~d})$ conditioned } \\
\hline & $E_{o}\left(\mathrm{kV} \mathrm{mm}^{-1}\right)$ & $\beta$ & $E_{o}\left(\mathrm{kV} \mathrm{mm}^{-1}\right)$ & $\beta$ & $E_{o}\left(\mathrm{kV} \mathrm{mm}^{-1}\right)$ & $\beta$ \\
\hline CT1 & $458 \pm 18$ & 17.1 & $438 \pm 35$ & 7.1 & $399 \pm 35$ & 5.0 \\
\hline NS3 & $325 \pm 15$ & 10.4 & $167 \pm 9$ & 10.4 & $112 \pm 7$ & 8.1 \\
\hline NS8 & $354 \pm 30$ & 5.3 & $163 \pm 11$ & 8.1 & $110 \pm 8$ & 7.5 \\
\hline NS18 & $355 \pm 20$ & 10.2 & $175 \pm 9$ & 10.1 & $115 \pm 10$ & 6.2 \\
\hline
\end{tabular}


the valid point that water can serve as a plasticiser, thereby increasing the free volume in the polymer. A later study on XLPE found an analogous decrease in AC breakdown strength with increasing water content [32] and, therefore, the available data appear to support our findings of a progressive reduction in breakdown strength in wet conditioned polyethylene blends without nanofiller. Similarly, few reports have considered the breakdown strength of nanocomposites as a function of water content. Ma et al. [33] compared the effects of adding dry and wet $\mathrm{TiO}_{2}$ nanoparticles to LDPE; dried particles provided a composite with the same breakdown strength as the host material whilst the incorporation of wet particles resulted in a significant reduction in the breakdown strength. Hui et al. [34] considered the effect of moisture on a series of XLPE/silica nanocomposites and showed a progressive reduction in breakdown strength with increasing moisture content. Finally, Jaeverberg et al. [35] compared the breakdown strength of epoxy/alumina nanocomposites exposed to dry and humid conditions and found that humid conditions significantly reduced the breakdown strength. Thus, all the available work seems to indicate a reduction in the breakdown strength of nanocomposites that contain water, in agreement with our findings.

\section{CONCLUSION}

Three blends incorporating 10 wt. \% of a nanosilica functionalized with different functionalizing agents (with different alkyl chain lengths) were compared to a control blend prepared without nanosilica. Structurally, the introduction of the nanosilica had little influence on the matrix polymer, beyond a minor nucleating effect and, in all the systems considered, the primary particles of nanosilica were found to be present in a range of agglomerated states. While even prolonged immersion in water was found to have little effect on the unfilled polyethylene blend, all nanocomposites revealed evidence of accumulation of water at the nanoparticle/matrix interfaces. The quantity of interfacial water present and its dynamics were found to be critically dependent upon both the nano-particle surface chemistry and the conditioning imposed upon the specimen. While the DC breakdown strength of the control blend was only weakly affected by conditioning, in the nanocomposites, variations in water content were found to result in marked changes in breakdown performance.

A key feature of nanodielectrics concerns the variability seen in published results, even when these were obtained from nominally comparable systems. The results presented here provide a new explanation for such effects. Variations in nanoparticle surface chemistry, both as a result of manufacturing route and subsequent chemical treatment, combined with different storage regimes, will inevitable result in variations in the quantity and aggregation state of water molecules at nanoparticle/matrix interfaces. Even small variations in this, in turn, result in significant variations in electrical behavior. Here, we have considered only DC breakdown strength; the following paper considers water effects more generally.

\section{ACKNOWLEDGMENT}

The authors gratefully acknowledge the RCUK's Energy Programme for the financial support of this work through the Top \& Tail Transformation programme grant, EP/I031707/1 (http://www.topandtail.org.uk/).

\section{REFERENCES}

[1] J. Ueda, M. Samusawa, K. Kumagai, A. Ishida, S. Tanabe, 2014, "Recreating the Lycurgus effect from silver nanoparticles in solutions and in silica gel", J. Mater. Sci., Vol. 49, pp. 3299-3304, 2014.

[2] Y. Kojima, A. Usuki, M. Kawasumi, A. Okada, Y. Fukushima, T. Kurauchi, O. Kamigaito, 1993, "Mechanical properties of Nylon 6clay hybrid", J. Mater. Res., Vol. 8, pp. 1185-1189, 1993.

[3] Y. Kojima, A. Usaki, M. Kawasumi, A. Okada, T. Kurauchi, O. Kamigaito, "Sorption of water in Nylon 6-clay hybrid", J. App. Polym. Sci., Vol. 49, pp. 1259-1264, 1993.

[4] L. Huang, R. Zhan, Y. Lu, "Mechanical properties and crystallization behavior of polypropylene/nano-SiO2 composites", $J$. Reinf. Plast. Comp., Vol. 25, pp. 1001-1012, 2006.

[5] M. G. Veena, N. M. Renukappa, J. M. Raj, C. Ranganathaiah,, K. N. Shivakumar, "Characterization of Nanosilica-Filled Epoxy Composites for Electrical and Insulation Applications", J. Appl. Polym. Sci., Vol. 121, pp. 2752-2760, 2011.

[6] T. J. Lewis, "Nanometric Dielectrics", IEEE Trans. Diel. Electr. Insul., Vol. 1, pp. 812-825, 1994.

[7] T. Tanaka, M. Kozako, N. Fuse, Y. Ohki, "Proposal of a Multi-core Model for Polymer Nanocomposite Dielectrics", IEEE Trans. Diel. Electr. Insul., Vol. 12, pp. 669-681, 2005.

[8] S. L. Abd-El Messieh, N. N. Rozik, "Dielectric and morphological studies on polyester/nanosilica fume composites", J. Appl. Polym. Sci., Vol. 122, pp. 714-721, 2011

[9] D. M. Panaitescu, Z. Vuluga, P. V. Notingher, C. Nicolae, "The effect of poly[styrene-b-(ethylene-co-butylene)-b-styrene] on dielectric, thermal and morphological characteristics of polypropylene/silica nanocomposites", Polym. Eng. Sci., Vol. 53, pp. 2081-2092, 2013.

[10] M. Praeger, A. S. Vaughan, S. G. Swingler, 2013, "The breakdown strength and localized structure of polystyrene as a function of nanosilica fill fraction", In Proc. 2013 Int. Conf. Sol. Diel., pp. 863866,2013

[11] K. Y. Lau, A. S. Vaughan, G. Chen, I. L. Hosier, A. F. Holt, K. Y. Ching, "On the space charge and DC breakdown behavior of polyethylene/silica nanocomposites", IEEE Trans. Diel. Electr. Insul., Vol. 21, pp. 340-351, 2014.

[12] K. Y. Lau, A. S. Vaughan, G. Chen, I. L. Hosier, A. F. Holt, "On the dielectric response of silica-based polyethylene nanocomposites", $J$. Phys. D: Appl. Phys., Vol. 46, Art. No. 095303, 2013.

[13] J. I. Hong, L. S. Schadler, R. W. Siegel and E. Martensson, "Rescaled Electrical Properties of ZnO/Low Density Polyethylene Nanocomposites", Appl. Phys. Letts., Vol. 82, pp. 1956-1958, 2003.

[14] D. Ma, T. A. Hugener, R. W. Siegel, A. Christerson, E. Martenson, C. Önneby and L. S. Schadler, "Influence of nanoparticle surface modification on the electrical behavior of polyethylene nanocomposites", Nanotechnology, Vol. 16, pp. 724-731, 2005.

[15] G. Iyer, R. S. Gorur, R. Richer, A. Krivda, L. E. Schmidt, "Dielectric Properties of Epoxy based Nanocomposites for High Voltage Insulation”, IEEE Trans. Diel. Electr. Insul., Vol. 18, pp. 659-666, 2011.

[16] M. Roy, J. Keith Nelson, R. K. MacCrone, L. S. Schadler, "Candidate mechanisms controlling the electrical characteristics of silica/XLPE nanodielectrics", J. Mater. Sci., Vol. 42, pp. 37893799, 2007. 
[17] M. Roy, J. K. Nelson, R. K. MacCrone, L. S. Schadler, C. W. Reed, R. Keefe and W. Zenger, 2005, "Polymer Nanocomposite Dielectrics - The Role of the Interface", IEEE Trans. Diel. Electr. Insul., Vol. 12, pp. 629-643, 2005.

[18] L. Hui, J. K. Nelson, L. S. Schadler, "The influence of moisture on the electrical performance of XLPE/silica nanocomposites", In Proc. 2010 IEEE Int. Conf. Sol. Diel., pp. 305-308, 2010.

[19] K. Y. Lau, A. S. Vaughan, G. Chen, I. L. Hosier, A. F. Holt, "Absorption Current Behavior of Polyethylene/Silica Nanocomposites", J. Phys. Conf. Series, Vol. 472, pp. 24-25, 2013.

[20] J. Castellon, H. N. Nguyen, S. Agnel, A. Toureille, M. Fréchette, S. Savoie, A. Krivda and L.E. Schmidt, "Electrical Properties Analyzis of Micro and Nano Composite Epoxy Resin Materials", IEEE Trans. Diel. Electr. Insul., Vol. 18, pp. 651-658, 2011.

[21] A. Sami, M. F. Frechette, E. David and S. Savoie, "Water as a Digression Relative to the Dielectric Response in the Frequency Domain for Polymer Composites", In 2008 Ann Rep Conf Electr. Insul. Diel. Phen., pp. 501-504, 2008.

[22] M. Praeger, A. S. Vaughan, S. G. Swingler, "A dielectric spectroscopy study of the polystyrene/nanosilica model system", In Proc. 2013 IEEE Int. Conf. Sol. Diel., pp. 859-862, 2013.

[23] T. Tanaka, "Dielectric nanocomposites with insulating properties", IEEE Trans. Dielectr. Electr. Insul., Vol. 12, pp. 914-928, 2005.

[24] K. Y. Lau, A. S. Vaughan, G. Chen, I. L. Hosier, A. F. Holt, "On nanosilica surface functionalization using different aliphatic chain length silane coupling agents", In Proc. 2013 IEEE Int. Conf. Sol. Diel., pp. 896 - 899, 2013.

[25] L. J. Brown, R. C. D. Brown, R. Raja, "Heterogenisation of ketone catalysts within mesoporous supports for asymmetric epoxidation", RSC Adv., Vol. 3, pp. 843-850, 2013.

[26] I. L. Hosier, A. S. Vaughan and S. G. Swingler, "An investigation of the potential of ethylene vinyl acetate/polyethylene blends for use in recyclable high voltage cable insulation systems", J. Mat. Sci., Vol. 45, pp. 2747-2459, 2010.

[27] R. H. Olley and D. C. Bassett, "An improved permanganic etchant for polyolefins", Polymer, Vol. 23, pp. 1707-1710, 1982.

[28] J. K. Nelson, J. C. Fothergill, L. A. Dissado, and W. Peasgood, "Towards an understanding of nanometric dielectrics", IEEE Conf. Electr. Insul. Diel. Phen., pp. 295-298, 2002.

[29] R. C. Smith, C. Liang, M. Landry, J. K. Nelson, and L. S. Schadler, "The mechanisms leading to the useful electrical properties of polymer nanodielectrics", IEEE Trans. Diel. Electr. Insul., Vol. 15, pp. 187-196, 2008

[30] P. A. M. Steeman, F. H. J. Maurer, "An interlayer model for the complex dielectric constant of composites", Colloid Polym. Sci., Vol. 268, pp. 315-325, 1990.

[31] Q. Lei, S. Sheng, "Effect of temperature and humidity on electrical breakdown characteristics of low-density polyethylene film for the various stressing modes", In Proc. $2^{\text {nd }}$ Intern. Conf. Prop. Appl. Diel. Mater., pp. 590-593, 1988

[32] G. Gao, W. Kalkner, "Effect of water content and temperature on the electrical breakdown strength of XLPE-insulated cables", In Proc. $7^{\text {th }}$ Intern. Symp. High Volt. Engin., pp. 251-254, 1991.

[33] D. L. Ma, R. W. Siegel, J. I. Hong, L. S. Schadler, E. Martensson, C. Onneby, "Influence of nanoparticle surfaces on the electrical breakdown strength of nanoparticle-filled low-density polyethylene", J. Mat. Res., Vol. 19, 857-863, 2004.

[34] L. Hui, L. S. Schadler, J. Keith Nelson, "The Influence of Moisture on the Electrical Properties of Crosslinked Polyethylene/Silica Nanocomposites", IEEE Trans. Diel. Electr. Insul., Vol. 20, pp. 641653, 2013.

[35] N. Jaeverberg, B. Venkatesulu, H. Edin, H. Hillborg, "Prebreakdown Current and DC Breakdown Strength of Alumina-filled Poly(ethylene-co-butyl acrylate) Nanocomposites: Part I Breakdown Strength", IEEE Trans. Diel. Electr. Insul., Vol. 21, pp. 2127-2134, 2014.

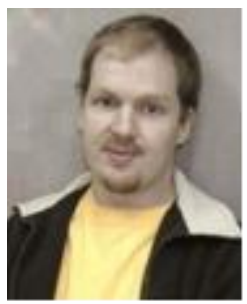

Ian Hosier was born in the UK in 1970. He received the B.Sc. degree in physics and the Ph.D. degree in polymer physics from The University of Reading, UK. He then went on with postdoctoral research at the same university, which involved extensive collaborations with institutions in Germany and Japan. In 2001 he moved to Florida State University in the USA before returning to the UK to take a research post at the University of Southampton.

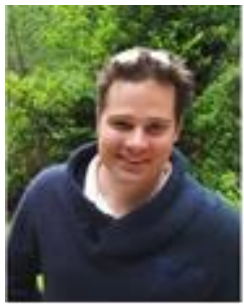

Matthew Praeger has an M.Phys degree in Physics with Laser Science and received a Ph.D for Laser Generation of X-rays, both from the University of Southampton. He subsequently worked at the Optoelectronics Research Centre before moving to the Electrical and Electronic Engineering group where his research has included electrical phenomena such as dielectrophoresis and nanocomposite materials for dielectric applications.

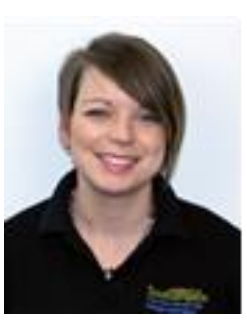

Alex Holt graduated from the University of Southampton with M.Chem. and Ph.D degrees in chemistry. After completing her PhD, she went on to works on a multidisciplinary project involving corrosion of copper and silver in transformers which utilizes a range of analytical techniques.

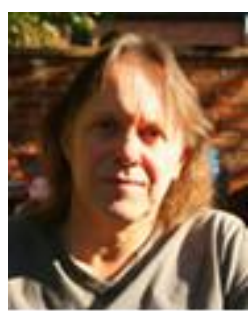

Alun Vaughan (SM'07) has a B.Sc. degree in chemical physics and a Ph.D. degree in polymer physics. After working at the UK's Central Electricity Research Laboratories and spending a period as an academic at The University of Reading, he is now Professor of Dielectric Materials and Head of the Electronics and Electrical Engineering research group at the University of Southampton. He is a former chair of The Dielectrics Group of the Institute of Physics, a Fellow of the Institute of Physics and a Fellow of the IET.

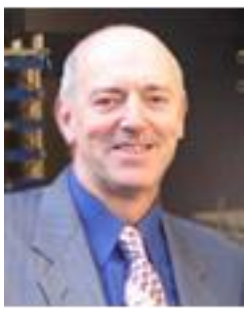

Steve Swingler received the B.A. and $\mathrm{Ph} . \mathrm{D}$. degrees in physics from the University of Lancaster. In 1976 he was appointed Pirelli Postdoctoral Research Fellow at Southampton University, investigating dielectrics for superconducting and extruded dc cables. He joined the Central Electricity Research Laboratories at Leatherhead in 1979 working mainly on novel cable systems and insulating materials. In 1990 he transferred to the National Grid Company, becoming Cables Manager and then Company Undergrounding Adviser. In 2003 he joined the School of Electronics \& Computer Science at Southampton University as Professor in Transmission Asset Engineering. He is the UK Member of CIGRE Study Committee B1 (High Voltage Cables). 